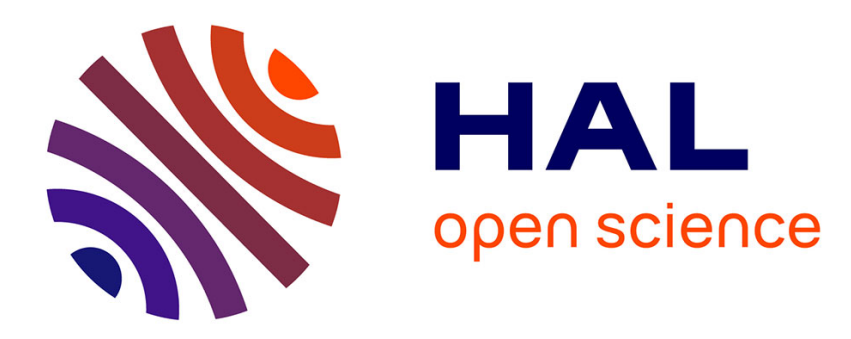

\title{
Entre les langues: l'être traduit du langage et la portée intraduite des mots
}

\author{
Catherine Cyssau
}

\section{To cite this version:}

Catherine Cyssau. Entre les langues: l'être traduit du langage et la portée intraduite des mots. Recherches en psychanalyse, 2005, Langues et traduction, 2 (4), pp.103-111. 10.3917/rep.004.0103 . hal-01502925

\section{HAL Id: hal-01502925 \\ https://hal.science/hal-01502925}

Submitted on 9 Apr 2017

HAL is a multi-disciplinary open access archive for the deposit and dissemination of scientific research documents, whether they are published or not. The documents may come from teaching and research institutions in France or abroad, or from public or private research centers.
L'archive ouverte pluridisciplinaire HAL, est destinée au dépôt et à la diffusion de documents scientifiques de niveau recherche, publiés ou non, émanant des établissements d'enseignement et de recherche français ou étrangers, des laboratoires publics ou privés. 


\section{ENTRE LES LANGUES: L'ÊTRE TRADUIT DU LANGAGE ET LA PORTÉE INTRADUITE DES MOTS}

Catherine Cyssau

Association Recherches en psychanalyse | Recherches en psychanalyse »

2005/2 n 4 | pages 103 à 111

ISSN $1767-5448$

ISBN 284795063X

Article disponible en ligne à l'adresse :

http://www.cairn.info/revue-recherches-en-psychanalyse-2005-2-page-103.htm

\section{Pour citer cet article :}

Catherine Cyssau, «Entre les langues: l'être traduit du langage et la portée intraduite des mots », Recherches en psychanalyse 2005/2 (n ${ }^{\circ}$ ), p. 103-111. DOI 10.3917/rep.004.0103

Distribution électronique Cairn.info pour Association Recherches en psychanalyse.

(C) Association Recherches en psychanalyse. Tous droits réservés pour tous pays.

La reproduction ou représentation de cet article, notamment par photocopie, n'est autorisée que dans les limites des conditions générales d'utilisation du site ou, le cas échéant, des conditions générales de la licence souscrite par votre établissement. Toute autre reproduction ou représentation, en tout ou partie, sous quelque forme et de quelque manière que ce soit, est interdite sauf accord préalable et écrit de l'éditeur, en dehors des cas prévus par la législation en vigueur en France. Il est précisé que son stockage dans une base de données est également interdit. 


\title{
Entre les langues : l'être traduit du langage et la portée intraduite des mots
}

\author{
Catherine Cyssau
}

«On ne traduit pas, on est traduit ${ }^{1}$. C'est le poète traducteur qui le dit héroïquement. Cette soumission à se laisser traduire par tout ce qui modifie l'être en puissance est héroïquement humble. Elle accepte de se prêter sensiblement à la portée intraduite des restes du temps, intraduite et intraductible terme à terme. Entre les langues, se tient tout ce qui reste : ici puise notre capacité à laisser venir du changement sa part d'angoisse, de négatif et d'absence. Mais sans «magie négative ${ }^{2}$. La soumission requise suppose une liberté qui n'est pas égoïste en ce qu'elle échappe à la contention sado-masochiste. La maîtrise, générée par les pulsions partielles, a déjà dû renoncer à son dernier office de maintenance du moi. C'est ainsi que l'être au fondement du subjectif, trouve son tissu du contact avec l'intraduit: celui-ci fonde le texte d'une langue dans sa ressource pulsionnelle et dans ses liens socio-affectifs. Ce fond ou ce reste intraduisible est ce qui rend l'être humain susceptible d'aimer, authentiquement d'être.

Pour Janine Altounian, «tout traducteur qui occulte le lieu de la perte en ne percevant pas ce reste échappant à la traductabilité, se comporte vis-à-vis du texte transmis comme un héritier qui effacerait la trace de ce qui n'a pu se dire dans son héritage, alors que pourtant c'est ce non-parlé qui a été fondateur de son existence. ${ }^{3}$

1. du Bouchet A. (1992), En hâte, p. 31.

2. Cf. chez Freud à propos de l'isolation obsessionnelle: le recours à une magie négative consiste, dans la névrose de contrainte, en un refoulement moteur : «enlever en soufflant dessus », Inhibition, symptôme, angoisse, Euvres complètes, tome 17, p. 236. Claude Barazer a attiré mon attention sur ce passage, lors d'une conférence à l'A.P.F. prononcée le 28 mai 2005.

3. Altounian J. (2005), L'intraduisible, avant-propos, p. XVII ; et p. 5 note 3. 


\section{TRACES DE LANGUE}

Les traces de langue sont-elles des traces d'affect? Le sens n'est pas premier mais la trace, «non localisable, jamais circonscrite - vivante, puis soi », écrit André du Bouchet ${ }^{4}$. Traduire porte atteinte au matériau du sens, le mot prend de l'avance sur la signification qu'il revêtira. Et puis quand le mot est arrêté, c'est à nouveau la langue qui déborde le mot.

Ainsi, se situant toujours entre deux langues, à tout instant est atteint hors de la langue, la terre. Cette matière du «sous-sol innervé» est ce qui centre l'écartement d'une langue, avec les rudiments exclus, dans le partage d'inutilisable et de dénuement.

«Traduisant, quand il me sera arrivé de traduire, je serai chaque fois allé à la rencontre aussi bien de quelqu'un - vivant comme mort ou mort comme vivant, cela revient au même - que de ma propre langue jamais rejointe encore ${ }^{5}$.

Quels sont ces restes de terre au bout des doigts ? Ceux de la terre d'Arménie chez Janine Altounian, qu'un flacon d'huile de rose ramasse à ses odeurs précieuses, terribles, nuancier émotif répétant un reste de réalité avec l'objet survivant du quotidien qui n'a pas disparu des gestes de mémoire ${ }^{6}$ ? Ou est-ce un résidu de parole auquel se résume, pour Freud, le défi du geste obsessionnel ? Ce dernier atteste d'une formation réactionnelle qui exclut la tendresse pour la couper de l'érotisme anal. C'est alors de la matière fécale qui laisse des résidus de saleté. Le rejet de l'érotisme anal frappe le langage devenu projectile réactionnel de défi ou de rabaissement, marque caractérielle contre-investissant la tendresse qui gît bafouée hors langue et sans adresse.

Comment traduire la trace de terre en donnant forme et temps aux mots, avec l'après-coup que le refoulement attaché à la maîtrise sphinctérienne a rendu possible ? Comment, pour parler, inclure cette confiance vouée à un autre - don relationnel, irrationnel ? Ce n'est plus, en ce cas, ni sujet ni interlocuteur, mais communauté du «on» qui se laisse traduire, inventer, recréer ou répéter. Les gestes libèrent mieux que les mots le sens de chacun en nous ${ }^{7}$.

Traduire fait subir à l'usage d'une langue un préjudice, à travers la matière qui, dans le mot, foule le sens illisible et non traduit.

4. du Bouchet A., ibid.

5. Ibid.

6. Altounian J. (2004), De quoi témoignent les mains des survivants ?, in J.-F. Chiantaretto (Ed), Témoignage et trauma. Implications psychanalytiques, pp. 27-62.

7. «Moi c'est aussi nous » dit M. Gribinski dans une conférence à l'A.P.F, le 28 mai 2005. Il fait remarquer l'absence de latence dans les situations totalitaires de régression psychopathologique ou collective, et l'enfermement narcissique qui prive d'une transmission possible du collectif au moi. 


\section{MATIÈRE ET MÉMOIRE}

En philosophie, il n'existe pas de distinction entre matériel et matière. Le matériel est ce qui se rapporte à la matière et s'oppose au formel et au spirituel. Epistémologiquement, la matière est opposée à la forme. Métaphysiquement, elle est opposée à l'esprit et prend le sens de substance qui constitue les corps.

Or la langue française dispose de trois termes différents qui n'ont pas le même emploi ni le même sens. D'autre part, dans la langue allemande, les notions de matière première ou brute (Rohstoff), ainsi que celle de matériel brut (Rohmaterial), sont utilisées par Freud. Nous nous en servirons pour approcher la notion de matière psychique, que nous différencions du matériau et du matériel psychiques ${ }^{8}$. La matière gagne à être reconsidérée du point de vue d'une mémoire qui, en ce cas, est moins conservatrice que transformative 9 . Ses traces coïncident avec telle matière intraductible de la transmission traumatique, et telle matière qui peut être sans langue dans la psychose.

\section{TRACES DE MÉMOIRE}

En 1900, dans L'interprétation du rêve, la matière est appelée au titre de «matière première » ${ }^{10}$, aux côtés du matériau infantile de l'inconscient et du matériel du manifeste, des souvenirs d'enfance, de la remémoration. La trace mnésique des matières premières, altérables et mobiles, se sépare de la conservation des traces mnésiques du matériau inconscient. Freud est pris dans un paradoxe où d'une part, les traces mnésiques constituent le frayage des représentations-choses dans l'inconscient, mais d'autre part, le matériel brut de la trace mnésique n'appartient pas au système inconscient qu'elle précède cependant. Seul le travail du rêve est alors susceptible de mobiliser et de transformer une matière première de la mémoire précessive à l'inconscient. La matière, nécessairement informe, de la trace mnésique est l'effet d'un premier transfert

8. Le terme Stoff signifie chez Freud, matériau (mais peut aussi se traduire par matériel, matière, substance). La matière première ou matière brute se dit Rohstoff. A côté de cet usage, se rencontre pour matière : les termes de Materie (dérivant du latin materia); pour matériel, le terme de Material, accompagné de celui de Rohmaterial, matériel brut, lequel rejoint d'une autre façon la question soulevée par la notion de matière première. (Je laisse de côté l'usage terminologique de Substanz également usité pour la matière substantielle).

9. C'est une hypothèse que j'ai avancée en 1995. Sa découverte est redevable à mes échanges avec André du Bouchet à partir de ses textes et de la place faite par son écriture au rapport distinctif entre matière et langue. Janine Altounian a conforté cette hypothèse et elle m'a apporté son éclairage traductif des termes en usage dans le texte freudien. Des discussions depuis 1995 avec René Roussillon sur la matière pulsionnelle, ainsi qu'avec Pierre Fédida sur la question du matériau et de la matière qu'il ne tenait pas particulièrement à différencier, avant 2000 et son éclairage de la régression, ont également compté. 
psychique dont la réalité n'est pas représentable et sans laquelle l'existence du matériau infantile ne serait pas figurable ${ }^{11}$.

Le terme de matériau évoque l'idée freudienne de fragments, de vestiges dont la forme architecturale défouie reste incomplète. Le matériau archéologique suppose un processus de formation psychique inconsciente, il se rapporte à des éléments mnésiques de la mémoire qui nécessitent une reformulation, il engage un travail de construction qui est seul capable d'établir des relations formelles aux parties manquantes et de traduire une vue d'ensemble auquel doit appartenir le matériau défoui. Le matériau rend compte, chez Freud, des processus associatifs primaires dans le système inconscient. Il peut être rapporté à la notion d'infantile, d'emblée sexuel et effet du refoulement. L'inconscient proprement-dit, constitué par les deux refoulements originaire et après-coup, reste le système où s'inscrit une conservation infantile de la mémoire susceptible de se construire en récit fantasmatique et fictionnel.

J'ai proposé de considérer l'existence d'un lieu Pré-Ics correspondant à la trace mnésique de perception que Freud représente, dans son schéma de l'appareil psychique, par les systèmes $S$. Il se situe dans le schéma régrédient du rêve, entre la perception hallucinatoire et le refoulement originaire qui clôture, en amont, le système Ics. Dans la topique freudienne, il appartient au matériel brut des systèmes $S$ pré-inconscients et actuels - composés de traces mnésiques de perception $(S)$ et de restes diurnes - d'indiquer la puissance transformante de la mémoire. Elle évoque la matière de la terre au sens où la rencontre André $\mathrm{du}$ Bouchet, ou encore la notion de lieu et de site telle que la conçoit Pierre Fédida ${ }^{12}$.

\section{MATIÈRE CORPORELLE, MATIÈRE SENSIBLE}

En 1915, le terme de matière est assimilée à la substance, «ce dont une chose est faite », à la «matière brute», inorganisée et non vivante, que par corrélation étymologique Freud associe avec la mère (materia, mater). La matière renvoie à la nature du terrain où la chose vaut pour le nom. Dans la nomination de Madère, l'importance des forêts lors de la découverte de l'île lui valut de s'appeler ainsi : en portugais madeira signifie bois.

«Vous reconnaissez que madeira n'est rien d'autre que le mot latin à peine modifié de materia, qui à son tour signifie matière en général [Stoff]. Or mateira

10. Je garde l'ancienne traduction par matière première bien que le terme usité chez Freud soit celui de Rohmaterial, matériel brut (et non de Rohstoff). Cf. L'interprétation du rêve (1900), O.C.t. 4, p. 592 : où à propos des systèmes $S$, il est question «des éléments du matériel mnésique brut»; et p. 597 : toujours à propos des systèmes $S$, «l'agencement des pensées du rêve est dissous, lors de la régression, en son matériel brut.»

11. Cyssau C. (1995), pp. 246-248.

12. P. Fédida (1994) (1989). 
est dérivé de mater, mère. La matière [Stoff] dont quelque chose est faite est pour ainsi dire sa part maternelle. ${ }^{13}$

La certitude matérielle de la mère, par opposition à la nomination symbolique du père repose, en ses fondements, si l'on suit le détour de madeira, sur la certitude sensorielle de la matière maternelle. Dans cette approche de la matière, le lien entre la matière première des traces mnésiques de perception et la matière corporelle de leur expérience sensorielle et pulsionnelle est indiqué. Il existe, certes, une matérialité sensible (de materia), mais la matière (Stoff) n'est pas dans un rapport fond/forme qui la ferait glisser de la substance matérielle au matériau construit par une représentation. Le lien que Freud effectue entre matière et mère est relatif à la source et à l'origine. Les substances corporelles ont participé à la matérialité sensible et au mouvement sensoriel et moteur dont se détache la matière première psychique. Celle-ci n'est donc pas exclusivement d'essence maternelle, si l'on tient compte de la part d'investissement sensoriel et pulsionnel qui la caractérise. Le matériau successif n'est pas originaire et n'est pas synonyme de matière ${ }^{14}$.

Cependant, lorsque le refoulé est mal assuré, lorsque la transitionnalité n'est pas suffisante pour permettre l'association représentation et affect, qui est le propre d'une intériorisation de l'inconscient, la matière peut venir en place du matériau psychique défaillant. La précarité des refoulements, dans les fonctionnements limites, positive alors le recours enfantin à une matérialité maternelle de la matière. Cette sollicitation narcissique fait risquer à l'entreprise transférentielle analytique de manquer «les réseaux sexuels de l'CEdipe» et «les axes fondateurs de la sexualité » qui ne se confondent pas impunément avec les revendications infantiles et narcissiques, comme le remarque Catherine Chabert ${ }^{15}$.

La non-distinction de la matière et du matériau infantile peut faire perdre de vue la problématique de telles pathologies où le sexuel est d'essence maternelle et sensorielle et n'a justement pas réussi sa transformation transitionnelle et transférentielle en refoulé fantasmatique. La psychothérapie des états-limites, suppose-t-elle, en ces cas, comme l'a pensé Pierre Fédida, de pouvoir accueillir

13. Trad. de P. Fédida (2000), p. 9. \& S. Freud (1915-1917), Leçons d'introduction à la psychanalyse, $O C$, t. 14, pp. 164-165.

14. Je me sépare du point de vue opposé de Pierre Fédida, lequel configure sur le même plan l'usage freudien de Materie-materia, de Substanz et de Stoff, dont il fait un matériau orginaire de construction: «La matière - materia - que le terme allemand de Stoff permet de bien entendre comme substance constitutive et donc comme matériau, est originaire en latin «le bois de construction » et donc littéralement ce bois qui est le matériau de construction » (2000), p. 9 .

15. Cf. Chabert C., Les fonctionnements limites : quelles limites ?, in J. André (Ed) (1999), pp. 104-105 : «Le danger ou l'écueil... réside dans le piège tendu par un discours... Où la dépendance se confond avec le maternel, où la désexualisation apparente masque la violence pulsionnelle, où les positions « infantiles » manifestes semblent reléguer, dans un ailleurs improbable, les mouvements d'amour et de haine engagés dans les réseaux sexuels de l'Edipe. Il faut donc maintenir les axes fondateurs de la sexualité, ne pas en négliger les supports pulsionnels pour éviter l'impasse ...d'une quête d'amour narcissique.» 
la matière psychique sous la référence de la «substance sexuelle » et chimique, telle que le rêve de l'injection à Irma propose sa «solution» ${ }^{16}$ ?

\section{MATIÈRE PULSIONNELLE TRANSFORMATIVE}

Avec l'Au-delà du principe de plaisir, Freud envisage, en 1920, la perspective d'une matière pulsionnelle. Le système $P c$-Cs est imaginé en feuillet, doté d'une couche superficielle de matière inorganique et non vivante qui le protège des stimuli externes. Freud lui oppose des pulsions organiques autoconservatrices. Celles-ci visent la régression et le retour vers la mort, mais se voient poussées à l'adaptation par la contrainte de la réalité qui est liée à l'histoire de la Terre. Les pulsions d'auto-conservation caractérisent principalement le moi, lequel est également alimenté des pulsions sexuelles narcissiques ${ }^{17}$. Freud, dans ce raisonnement proche du lamarckisme, distingue entre une matière non vivante originaire et superficielle et un matière vivante, biologique et organique :

«Les pulsions viennent de ce que la matière non douée de vie a pris vie et elles veulent réinstaurer l'état non doué de vie... C'était peut-être un processus d'un modèle analogue à cet autre qui plus tard a fait apparaître la conscience dans une certaine couche de la matière vivante. ${ }^{18}$

Cette métaphore freudienne de la matière annonce des voies pour penser le vivant au-delà de la séparation de l'inorganique et de l'organique que l'on retrouve dans les chemins désormais empruntés par la biologie moléculaire, la physique ou la chimie. Pour P. Levy, il n'est donc pas aberrant de penser que l'on puisse «passer par exemple, d'un élément de matière inorganique complexe à un élément de matière vivante ${ }^{19}$.

La matière pulsionnelle est explosive, elle fonctionne comme principe transformatif. L'émotion, l'empreinte affective, le langage, mais aussi les mouvements pulsionnels de la sexualité non narcissique vont servir au traitement de la matière brute: sa qualité psychique en fait un mixte d'éléments individuels et collectifs, d'éléments de soi et de l'environnement. La matière première psychique génère un double mouvement psychique, à la fois, au contact du monde extérieur et, à la fois, au contact du monde interne. L'influence de C. Darwin sur la rencontre pour Freud du concept de matière première et pulsionnelle apparaît alors, de façon assez évidente. «Transformer la matérialité brute du monde environnant » et « s'auto-transformer ${ }^{20}$ à ce contact avec

16. Fédida P., Un patient de rêve pour un psychanalyste ?, in J. André (Ed) (1999), p. 77.

17. Freud S. (1920), Au-delà du principe de plaisir, O.C. t. 15, p. 309.

18. Ibid, pp. 315-316.

19. Levy P. (1996), pp. 213-228.

20. Chouvier B. (1998), p. 104. 
la réalité rencontrent la perspective phylogénétique freudienne de l'acquisition adaptative des névroses de transfert et des compétences psychiques et techniques qu'elles développeront pour adapter le monde environnant. C'est encore de ce point de vue que Y. Gampel peut comparer la mémoire traumatique et ses restes transmis généalogiquement aux «résidus radioactifs... qui se frayerait un chemin dans notre psyché en touchant les trois espaces psychiques, à savoir l'intersubjectif (des liens, relations d'objet), l'intrasubjectif (l'individuel, le plus intime, pulsion et représentations de pulsions), et le trans-subjectif (contexte socio-culturel) $\gg .{ }^{21}$

\section{MATIÈRE ET MATÉRIAU DE LA LANGUE}

Entre les langues ${ }^{22}$, André du Bouchet évolue entre la langue de l'enfance, l'allemand d'Holderlin, celle des années d'université où exprimer «les idées en cours d'érosion », l'américain, l'intervalle inconnu du français, langue d'origine du patronyme, et «l'interdit de langue» qui est «le bruit du russe», langue proscrite et cependant privé entre ses parents. Entre ses quatre langues, une matière étrangère restant intraduite d'une personne à l'autre et d'une langue à l'autre porte le mouvement de traduire.

Pour traduire, il sera nécessaire d' «entrer dans la langue» et pour entrer dans la langue à traduire, accepter de se perdre. C'est dans l'écart de langue à langue, comme d'être à être, que l'effet traducteur d'autre, qui n'est pas toujours, du reste, traductible terme à terme, précipite à la rencontre de la langue inconnue, en même temps qu'elle découvre l'écart «de ma propre langue jamais rejointe encore ». C'est ce qui reste à traduire dans sa langue, dont dépend la traduction. C'est ce qui la déborde et que la traduction, comme l'intimité de l'étranger vient rencontrer - le russe, bruit chaleureux, hostile, sexuel, fou. La traduction d'une langue à l'autre comporte une exclusion de langue, ici le bruit du russe, sous-sol inhumain et intime. «Jamais, dit-il, je n'ai été assuré du français. Où j'ai traduit, l'original a disparu, il est devant moi ».

La «matière sans langue » fait la langue et la meut, au-delà de son matériau signifiant/signifié ; elle se trouve en coïncidence avec la disparition du mot et du sens qui n'obtient pas la destination d'une signification, ou qui accepte, dans la déportation de la traduction, de s'en défaire.

«... de l'autre côté de la prairie, une matière humaine malmenée - matière sans langue - hulule » ${ }^{23}$. André du Bouchet venait de croiser un groupe d'enfants fous d'une institution psychiatrique voisine. Cette intruse perception d'une

21. Gampel Y., Penser la mémoire impensable de l'extermination, in J. Gillibert et P. Wilgowicz (Ed) L'ange exterminateur, Cerisy, pp. 173-175, citée par J. Altounian (2005), p. XIII note 1 .

22. du Bouchet A. (1992), En hâte.

23. du Bouchet A. (1980). 
matière malgré tout humaine au hululement mutique fait toucher à un point de surdité ${ }^{24}$. Est-ce en cette cassure qu'il faut situer un seuil d'inhumain de l'humain à franchir, une faille de l'effondrement et le lieu de transmission de la part du nous collectif qui me traverse ? L'assourdissant hululement où s'encolle la psychose, mais aussi l'enchantement séducteur qui cloue Ulysse à son mât, est faute d'après-coup, de refoulement opérant, et de latence. Il y a, dans cette non-rencontre de la masse psychotique ou de l'effet de masse séducteur ${ }^{25}$, une adhésion ainsi qu'une adhésivité «à la prairie». La «matière sans langue » en ces cas, se voit dépourvue d'amnésie comme de mémoire historisante.

Matière de l'interlocuteur, "non matériau, si la poésie tient aussi à l'inutilisable» ${ }^{26}$. Pour du Bouchet: «La vérité de dénuement ne se soutient pas ». Traduire touche à cela. Le matériau du langage se trouve, ainsi, défait de sa prescription et, momentanément, coupé d'un sens significatif. Il retourne à l'intraduit d'une «matière sans langue » ${ }^{27}$. Une cassure, une disparition conduit la langue froide à cette «matière de la fusion ». L'emploi des termes chez du Bouchet n'est pas de pure métaphore, il a un ancrage réel précis et perceptif. Le magma en fusion, dont notre Terre est faite, modifie les traces des strates géologiques, sans cesse chimiquement contrariées par des apports étrangers de nouveaux produits magmatiques qui perturbent et déséquilibrent leurs stratifications. De même, la «matière de la fusion » d'une langue se défait de forme et de sens, détachant «à travers le mot, la matière même du mot comme nontraduit».

André du Bouchet a été un traducteur apprécié de l'œuvre de Lacan en anglais. Rejoignant Freud, à propos des souvenirs écrans, à propos de la «perte de mémoire », il fait de l'écriture une œuvre de traduction de langage qui n'est pas identique à celle de la parole. Ecrire en traduisant, traduire en écrivant, «aiguillé par la dessaisie, et dans le même temps, sur le passage à vide, aimanté par l'or d'un inaccessible espoir. L'or, ou mieux - étranger à la valeur - le blanc »; blanc du papier dont on sait la fonction incontournable dans ses poèmes. Pour l'écriture, la matière perdue au souvenir, muette, immémoriale et amnésique, touche au commun, au «on », à la résurgence du blanc, elle s'en détache, s'en décale durant la marche. Papier comme chemin ou montagne, pour le poète, resteront toujours en avance sur la capacité de l'écriture d'un mot à traduire le retard d'un mouvement de langue atteinte, cette fois, d'amnésie.

Catherine CYSSAU

6 impasse Marie Blanche

75018 Paris

24. dont parle autrement J.-M. Vives à propos de la voix (2005).

25. Cf. Fédida P. (1983).

26. Dédicace d'André du Bouchet à son livre Matière de l'interlocuteur, 1992. Les citations suivantes se rapportent à ce texte pp. 34-41.

27. du Bouchet A. (1980). 


\section{BIBLIOGRAPHIE}

ALTOUNIAN J. (2005), L'intraduisible. Deuil, mémoire, transmission, Paris, Dunod.

ALTOUNIAN J. (2004), De quoi témoignent les mains des survivants ?, in J.-F. Chiantaretto

(Ed), Témoignage et trauma. Implications psychanalytiques, Paris, Dunod, pp. 27-62.

ANDRÉ J. (Ed) (1999), Les états limites, Paris, P.U.F.

DU BOUCHET A. (1980), Rapides, Paris, Hachette POL.

DU BOUCHET A. (1992), Matière de l'interlocuteur, Fata Morgana.

DU BOUCHET A. (1992), En hâte, L'âne, Le magazine freudien, avril-juin, pp. 31-32.

CHOUVIER B. (Ed) (1998), Matière à symbolisation, Lausanne, Delachaux et Niestlé.

CYSSAU C. (1995), Au lieu du geste, Paris, P.U.F.

FÉDIDA P. (1983), L'effet de masse, Furor, Genève, pp. 5-15.

FÉDIDA P. (1989), Théorie des lieux, Psychanalyse à l'université, t. 14 n 53 pp. 3-16 et $n^{\circ} 56$ pp. 3-18.

FÉDIDA P. (1994), Le site de l'étranger, Paris, P.U.F., 1994.

FÉDIDA P. (2000), Par où commence le corps humain, retour sur la régresion, Paris, P.U.F.

FREUD S. (1900), L'interprétation du rêve, CEuvres complètes t. IV, P.U.F.

FREUD S. (1916-17), Leçons d'introduction à la psychanalyse, Euvres Complètes, t. 14, Paris, P.U.F.

FREUD S. (1920), Au-delà du principe de plaisir, CEuvres complètes, t. 15, Paris, P.U.F.

FREUD S. (1925), Inhibition, symptôme, angoisse, CEuvres complètes, t. 17, Paris, P.U.F.

LEVY P. (1996), Sur la connaissance de la matière inorganique, L'inactuel, La matière, Paris, Calmann Levy, n 5, pp. 213-228.

VIVES J.-M. (2005), Pour introduire la question du point sourd, Psychologie clinique, $\mathrm{n}^{\circ} 19$, pp. 9-20.

Catherine Cyssau - Entre les langues : l'être traduit du langage et la portée intraduite des mots

Résumé: La traduction modifie l'être, elle conduit à repenser les transformations de la mémoire, ses traces, sa part pulsionnelle et sensible, collective et individuelle. La langue, l'écriture poétique et la transmission composent avec ce qui va toujours rester intraduit. Cette question confronte la matière de mémoire avec les transmissions collectives, traumatiques, et le destin du langage dans la psychose.

Mots-clés : Traduction - Langue - Matière - Traces - Mémoire.

Catherine Cyssau - Between Languages :The Human Being Translated in Language and the Untranslated Power of Words

Summary : Translation changes the being, it leads us to rethink memory-transformations, memory traces, those parts of memory that are instinctive and sensitive, collective and individual. Language, poetic writing and transmission have to make do with what will always necessarily remain untranslated. This question confronts the material of memory with collective and traumatic transmissions, and the fate of language in psychosis.

Key-words: Translation - Language - Material - Traces - Memory. 